\title{
Is three greater than five: The relation between physical and semantic size in comparison tasks
}

\author{
AVISHAI HENIK and JOSEPH TZELGOV \\ Department of Behavioral Sciences, Ben-Gurion University of the Negev, Beer-Sheva 84120, Israel
}

\begin{abstract}
In this study, subjects were asked to judge which of two digits (e.g., 3 5) was larger either in physical or in numerical size. Reaction times were facilitated when the irrelevant dimension was congruent with the relevant dimension and were inhibited when the two were incongruent (size congruity effect). Although judgments based on physical size were faster, their speed was affected by the numerical distance between the members of the digit pair, indicating that numerical distance is automatically computed even when it is irrelevant to the comparative judgment being required by the task. This finding argues for parallel processing of physical and semantic information in this task.
\end{abstract}

When two multidimensional stimuli are compared along one dimension, irrelevant aspects of the stimuli may intrude and influence the decision. This suggests an inability to ignore or filter information even when it is irrelevant. It is important to know how the filtering of an irrelevant dimension depends upon the similarity of its processing to that required for the relevant dimension. Some dimensions are physical (e.g., size, color); others are based on stored semantic information (e.g., numerical value). Mental comparisons often involve both physical and semantic dimensions. Manipulation of both physical and semantic dimensions as relevant or irrelevant to the comparison should help define how they interact. Furthermore, when a dimension such as size or value is being processed, the information extracted can be crude (e.g.; large or small) or finegrained. We wish to find out how detailed the processing of the irrelevant dimension is. In order to study these questions, we manipulated both the numerical and physical size of digits in comparative judgment tasks.

The stimuli in our study were pairs of Arabic numerals. We manipulated the physical size (i.e., height) and the semantic magnitude (i.e., numerical value) of the stimuli. Each pair of digits could be characterized as congruent, incongruent, or neutral. In a congruent pair, the physically larger digit was also numerically larger (e.g., 53 ). In an incongruent pair, the physically larger digit was numerically smaller (e.g., 5 3). Neutral stimuli were those in which the two digits were the same on the irrelevant dimension (e.g., the pair 53 can be

The order of authorship was determined randomly. We would like to thank L. Bechor, O. Tramer, and S. Tramer for their help in running the experiments, $O$. Argaman for help in preparing the manuscript, and F. J. Friedrich and two anonymous reviewers for their helpful comments. We are grateful to M. I. Posner for his extensive help in preparing the manuscript. Requests for reprints should be sent to Avishai Henik, Department of Behavioral Sciences, Ben Gurion University of the Negev, Beer-Sheva 84120 , Israel. used for numerical comparison, since the two digits differ in their numerical values but are the same in their physical sizes). The subjects were asked to decide which digit was larger according to either physical size or numerical value.

When subjects are asked to compare the numerical size of two simultaneously presented digits, incongruent physical size interferes with performance (Besner \& Coltheart, 1979; Hinrichs, Note 1; Yurko \& Hinrichs, Note 2). ${ }^{1}$ The general phenomenon of interference by physical size in semantic magnitude comparisons has been called the "size congruity effect" by Banks and Flora (1977), who argue that the effect is produced at an early stage of processing. Paivio (1975) found a size congruity effect using pictures of different sizes. His results thus accord with a model in which physical differences between the two elements of the pair have an effect on comparison of the semantic dimension.

But what will happen if the task is changed, so that the subjects are asked to compare two digits on the basis of their physical size? If physical and numerical differences are determined in serial order, there should be no influence of numerical size on physical judgments, provided that the former is a slower process. In contrast, a parallel model would allow some influence of numerical magnitude on physical judgment. The pattern of influence, however, should depend upon the facets of numerical information that are extracted in parallel with the physical judgment. Suppose that only crude descriptions of the digits' numerical dimension (e.g., "large" or "small") are determined in parallel with the physical judgment. This information would be sufficient to produce an effect of congruence. That is, subjects would be slower when the physically large digit is smaller in number than when the physically large digit is also large numerically. However, one would not expect an effect of the numerical distance between the digits on size comparisons, since the computation of this information involves relative rather than absolute numerical informa- 
tion. If the numerical information extracted in parallel with the physical judgment is more detailed, one may expect an effect of numerical distance on physical size comparisons. That is, the numerically distant pair of digits (e.g., 26) would have a larger influence than the numerically closer pair (e.g., 24). Thus the pattern of interference effects can serve to specify the type of operations on numerical information that occur in parallel with the physical computation.

In order to look at the details of the influence exerted by the irrelevant variable in a comparative judgment task, we performed two experiments. In the first experiment, we examined whether either physical or semantic dimensions can influence performance when irrelevant to a task based on the other dimension. In this experiment, we manipulated congruence and type of comparison (physical comparisons vs. semantic comparisons). In the second experiment, we also manipulated the semantic distance between the two compared digits.

\section{EXPERIMENT 1}

On each trial, subjects were exposed to pairs of digits. They were asked to decide which digit in each pair was larger on the relevant dimension. In one session, the relevant dimension was semantic (numerical) size, and in the other session, the relevant dimension was physical size. Three types of stimuli were used. Congruent (e.g., 5 3), neutral (e.g., 53 for numerical comparisons, 33 for physical comparisons), and incongruent (e.g., 5 3). Our first question was whether the size congruity effect would appear for both physical and numerical size as relevant dimensions. If physical information is processed before the semantic information, we should find a size congruity effect only when semantic size is the relevant dimension. If, however, the two dimensions are processed in parallel (at the same or different rates), a size congruity effect is expected for both dimensions.

Two alternative predictions about the direction of the size congruity effect are possible. A cost-benefit analysis would predict fastest reaction time (RT) for congruent stimuli (e.g., 53 ) and slowest for incongruent stimuli (e.g., 5 3). An intermediate response time should be expected for neutral stimuli (e.g., 53 ). An alternative prediction was recently implied by Dixon and Just (1978) in their normalization model. According to this model, the subject must nullify irrelevant differences between the items to be compared. This end is achieved by mentally equating the items on the irrelevant dimension before comparing them on the relevant dimension. Thus, when the subject is asked to decide which of the two digits presented is numerically larger, the response should be faster when the two digits are of the same physical size (e.g., 53 ) than when the two digits differ in physical size, be they congruent (e.g., 53 ) or incongruent (e.g., 53 ) stimuli. This prediction follows from the assumption that in the last two conditions, the subject has to perform normalization on the physical size (the irrelevant dimension) and that this process is timeconsuming.

It may be easier to conceive a normalization process for an irrelevant physical dimension, especially for physical size, than for a semantic, more abstract dimension. However, the claims for an "internal psychophysical judgment" performed during comparisons of animal names (Moyer, 1973; Moyer, Bradley, Sorensen, Whiting, \& Mansfield, 1978) lend credit to the suggestion that a normalization process may be applied to semantic as well as physical material. The use of semantic size as an irrelevant dimension should address this issue. Thus, in the present experiment, we contrast the prediction of the normalization model with the prediction of a cost-benefit analysis for the size congruity effect. Moreover, this test is carried out for both physically and numerically irrelevant dimensions.

\section{Method}

Materials. Each subject performed two kinds of comparisons. In one, the relevant dimension was physical size, and in the other, numerical value. Eighty stimuli were prepared, each consisting of a pair of digits. The 80 stimuli included 20 congruent, 20 incongruent, and 40 neutral pairs. A congruent stimulus was defined as a pair of digits in which a given digit is larger on both the relevant and irrelevant dimensions. A neutral stimulus was defined as a pair of digits that differ only on the relevant dimension. An incongruent stimulus was defined as a pair of digits in which a given digit is simultaneously larger on one dimension and smaller on the other. The digits 2-8 were used. Each digit was paired with itself (and served as a neutral stimulus for physical comparison) or it was paired with a different digit that was numerically larger or smaller by 2 units. The two digits in each pair could be of the same physical size (and in this case the pair served as neutral for numerical comparisons) or could differ in height (physical dimension) by $1 \mathrm{~mm}$. The possible heights of the digits were 4,5 , and $6 \mathrm{~mm}$. Examples of the stimuli for both comparisons are shown in Figure 1. Only the neutral stimuli were different in the two comparisons. A

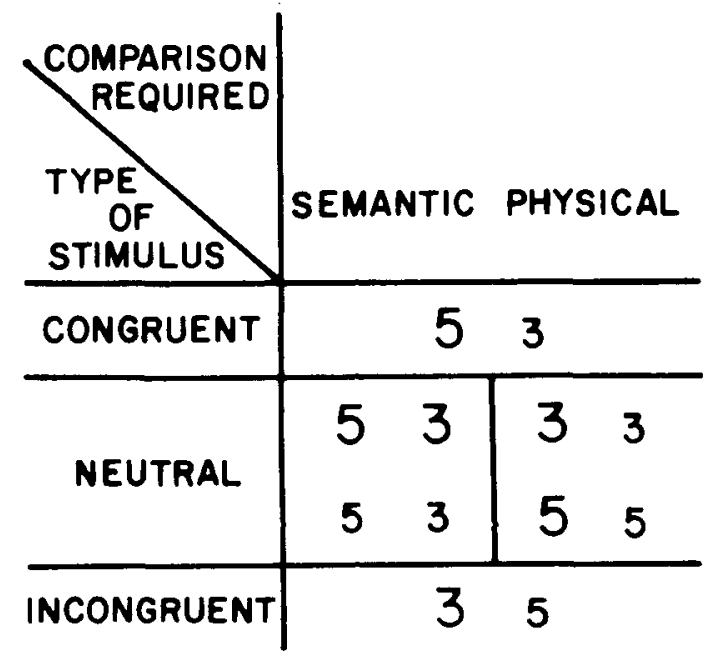

Figure 1. Examples of stimuli used in Experiment 1. For congruent and incongruent situations, the same stimuli served for both physical and semantic comparisons. 
stimulus display consisted of two digits that appeared centered around the fixation point. The center-to-center distance between two digits was $10 \mathrm{~mm}$. The digits used were Alphac 32 .

Within the set of stimuli prepared for each kind of comparison, each digit and each physical size appeared on both sides of the visual field equal numbers of times. The equipment consisted of a Gerbrands Harvard three-field tachistoscope and a Monsanto HT 8510 counter/timer. The display scope was $80 \mathrm{~cm}$ from the subjects' eyes.

Design. The experiment consisted of two sessions that differed in the relevant dimension for the required comparisons. The variables manipulated were: relevant dimension (physical vs. semantic), order of sessions (physical first vs. semantic first), congruence (congruent, incongruent, neutral). Thus, we have a 2 by 2 by 3 factorial design. Order of sessions was the only between-subjects variable.

Procedure. The subject's task was to decide which of the two digits in a given display was larger. Each subject participated in two sessions on 2 different days. In one session, "larger" was defined by physical size; in the other, it was defined by numerical (semantic) value. The stimuli in each session were presented in a random order. Half of the subjects performed physical comparisons on the first session, and the other half performed numerical comparisons on the first session. Each session began with eight practice trials. The subjects were asked to respond as quickly as possible but to avoid errors. The subjects indicated their choice by pressing one of two levers on the same side as the chosen item. Each trial began with a fixation point presented for $100 \mathrm{msec}$. The fixation point was immediately followed by a pair of digits that remained in view until the subject's keypress. This stopped the timer and offset the stimulus. After each trial, accuracy feedback was given.

Subjects. Twenty-six students served as subjects. Their participation was in partial fulfillment of course requirements.

\section{Results}

Error rates were low in the present experiment. In the numerical comparison condition, there were $.19 \%$ errors in congnuent trials, $2.5 \%$ in incongruent trials, and $.48 \%$ in neutral trials. In the physical comparison condition, there were $.19 \%$ errors in congruent trials, $.78 \%$ in the incongruent trials, and $.01 \%$ in the neutral trials. These error percentages fit in rather nicely with the RT data, which are discussed in the following section. The only exception is the order of neutral relative to congruent trials in the physical comparison condition.

The following analyses were performed on RT measures, that is, the time elapsed between stimulus onset and subject's response. Mean RTs for the various treatments are presented in Figure 2. The data were submitted to a three-way analysis of variance, with congruence and relevant dimension as within-subjects factors and order of sessions as a between-subjects factor. This analysis was performed on the means of correct trials for each subject in each experimental condition.

Collapsing over all other factors, physical comparisons were made $125 \mathrm{msec}$ faster than semantic comparisons $[F(1,24)=111.21, p<.001]$. The three levels of the congruence variable also differed significantly $[F(2,48)=89.65, p<.001]$, with mean RTs of 650 , 585 , and $548 \mathrm{msec}$ for incongruent, neutral, and con-
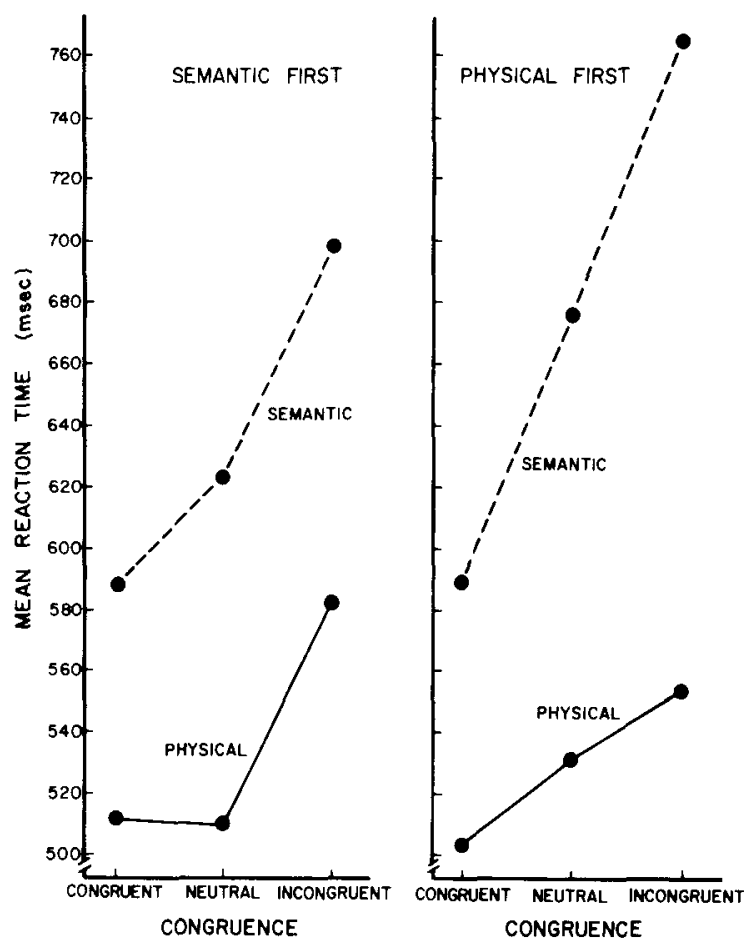

Figure 2. Mean reaction time in milliseconds as a function of relevant dimension, congruence, and order of sessions.

gruent pairs, respectively. However, the effect of congruence varied as a function of both relevant dimension and order of sessions $[F(2,48)=3.68, p<.05]$. This is also evidenced in the two first-order interactions, between congruence and relevant dimensions $[F(2,48)=14.32$, $\mathrm{p}<.001]$ and congruence and order of sessions $[F(2,48)=3.39, \mathrm{p}<.05]$.

The second session for each subject could have been influenced by the experience in the first session, since the relevant dimension in the first session acted as irrelevant in the second session. Thus, simple effects of interaction between congruence and relevant dimension were tested separately for each group of subjects. For subjects who based their decision on physical sizes in the first session, the interaction between congruence and relevant dimension was highly significant $[\mathrm{F}(2,48)=16.11$, $\mathrm{p}<.001]$. For those who made numerical comparisons in the first session, the effect was in the same direction but significant to a lower level $[F(2,48)=3.49$, $p<.05]$.

In the last part of the analysis, the means of the various congruence conditions were compared separately for each session and each relevant dimension via the Newman-Keuls technique (see Table 1). The difference between congruent and incongruent pairs of items was significant for both types of judgments. The differences between the neutral stimuli and the other two types of stimuli were more reliable for the semantic comparisons than for the physical comparisons. 
Table 1

Significance Levels of Newman-Keuls Tests on Pairs of Congruence Levels as a Function of Type of Comparison

\begin{tabular}{lccc}
\hline & \multicolumn{3}{c}{ Congruence Level } \\
\cline { 2 - 4 } $\begin{array}{c}\text { Type of } \\
\text { Comparison }\end{array}$ & $\begin{array}{c}\text { Congruent vs. } \\
\text { Incongruent }\end{array}$ & $\begin{array}{c}\text { Congruent } \\
\text { vs. Neutral }\end{array}$ & $\begin{array}{c}\text { Incongruent } \\
\text { vs. Neutral }\end{array}$ \\
\hline & \multicolumn{3}{c}{ First Session } \\
Semantic & .01 & .05 & .05 \\
Physical & .01 & $*$ & $*$ \\
& & Second Session & \\
Semantic & .01 & .01 & .01 \\
Physical & .01 & $*$ & .01 \\
\hline
\end{tabular}

Note-In each case, $p<$ value given. $\quad$ *Nonsignificant.

\section{Discussion}

Let us summarize the results presented in the preceding section: (1) Physical comparisons take less time than semantic comparisons. (2) Congruence has an effect on performance. RTs for congruent tasks are always shorter than RTs for incongruent tasks. RTs for congruent tasks are shorter than RTs for neutral tasks for semantic comparisons only, whereas RTs for incongru. ent tasks are usually longer than RTs for neutral tasks. This last statement does not hold for first sessions in which comparisons are based upon the physical dimension. $^{2}$ (3) The interaction between congruence and relevant dimension seems to be stronger for subjects who performed physical comparisons in their first session.

We can conclude that irrelevant differences, regardless of their qualitative nature (physical or numerical), affect performance. This conclusion is particularly interesting in light of numerical comparisons being generally slower than physical comparisons. Thus, when comparisons are based upon physical size, the slower process (numerical) influences the faster (physical) one. This means that both these processes are executed in parallel. It is not possible, at this point, to claim that the numerical information is fully processed. However, it is reasonable to suggest that numerical processing ends up with, at least, absolute codes for each digit. That is, each digit is labeled as being either large or small. Moreover, the system notes the difference between the two digits on both the relevant and the irrelevant dimensions.

Earlier, we introduced two alternative predictions with respect to the direction of the size congruity effect. According to the cost-benefit analysis, the congruent condition will be faster than the neutral condition, since the benefits are gained from the correspondence between relevant and irrelevant dimensions. According to the normalization view, the neutral trials will be fastest, since they do not require normalization. The present results support the first suggestion rather than normalization. We found both facilitation and inhibition of RT as a result of the correspondence between the semantic and physical aspects of the stimulus. The directional influence of congruence on RT makes it difficult to apply the normalization model to such tasks.

A normalization hypothesis was considered by Holyoak (1977) with regard to size congruity of mental images. The results showed that subjects can normalize their images when instructed to do so. However, no evidence for normalization was found in the control group. The control subjects used imagery but did not receive specific instructions with respect to the comparison process. Moreover, even the experimental group showed effects of incongruency, similar to ours, over and above the normalization effects. Thus, it seems that normalization is a possible way of dealing with images but it is not the probable way, nor is it probable in comparative judgments of numbers. In the process of comparing two elements, the system notes the direction of the disparity between the two elements even for irrelevant dimensions. Does the system note the numerical distance between the two elements when this information is irrelevant to the task? The next experiment addresses this issue.

\section{EXPERIMENT 2}

We have found that the system notes irrelevant differences in parallel with the processing of relevant differences. An interesting question relates to the level of detail of such processing. Does irrelevant processing stop at the level of crude description of the stimuli or does the system process irrelevant information in a more detailed fashion? If the latter alternative is true, one could expect effects of irrelevant numerical distance on performance. A related issue is the relationship between the effects of congruence (size congruity effect) and the effect of numerical distance between the digits. These two issues are relevant to a model of comparative judgment tasks, suggested by Banks (1977).

Banks' (1977) model includes a number of consecutive stages. The function of the first stage is code generation. The possible codes are "large" (L) and "small" (S). If the two compared items are far apart on the continuum of judgment, it is probable that their codes will be different. However, if the two items are very close, an additional discrimination is required. This is done in the discrimination stage, which follows code generation. The next two stages deal with matching stimulus and instructional codes (third stage) and response selection (fourth stage). Banks suggests that the source of the size congruity effect is code generation, whereas the source of the distance effect (faster RTs with increased semantic distance between the compared elements) is the discrimination stage. When the two compared items are closer on the relevant dimension, it is more probable that an added time-consuming manipulation will occur and thus elevate response time. Banks argues that the two effects originate at different stages, 
since the two have additive effects on RT. This reasoning is based upon the additive factors notion, suggested by Sternberg (1969). This method assumes that the input is processed by successive stages, so that the output of one stage is fed into the next stage. Sternberg suggested that any two variables that affect different stages of processing would have additive effects on RT. Any two factors that affect the same stage would have an interactive effect on RT. Thus, finding no interaction between congruence and semantic distance hints at an additive relationship between the two stages affected by these variables. This result was found when subjects compared real-life sizes of drawings of animals (Banks, 1977). We tested the prediction of additivity by manipulating the relevant dimension, congruence, and numerical distance. The numerical comparisons will be a replication of Banks' experiment (with a different sort of stimuli); the physical comparison condition is new.

The question regarding the details of irrelevant processing is related to this model also. If we find that numerical distance has an effect on physical comparsons, it would suggest that detailed numerical processing is being made in parallel with physical processing. Such a finding would call into question the idea that features are processed by consecutive stages.

The present experiment was performed in the same paradigm used in Experiment 1. However, in the first experiment, semantic distance was fixed (2 semantic units), whereas in the second experiment, there were two semantic distances: 2 units (e.g., 3 5) and 4 units (e.g., 3 7).

\section{Method \\ Materials. Almost all aspects described in the Materials section of Experiment 1 apply here as well. However, three differences must be emphasized. (1) Stimuli having two digits that are different with regard to their numerical values con- sisted of the following pairs: $24,35,68$ for semantic distance of 2 units; $26,37,48$ for semantic distance of 4 units. (2) There were two physical differences: $1 \mathrm{~mm}$ (pairs with 4-and 5 -mm digits or pairs with 5-and 6-mm digits) and $2 \mathrm{~mm}$ (pairs with 4- and 6-mm digits). ${ }^{3}$ (3) Each congruence level comprised 36 stimuli (12 stimuli for each pairing of heights: $4-5 \mathrm{~mm}$, 5-6 mm, 4-6 mm).}

Design. The design was similar to the design of Experiment 1 , with the following exceptions: (1) We added a fourth variable, namely, semantic distance, which consisted of two levels as described in the Materials section, and (2) it was not possible to define neutral stimuli for physical comparison that were different for different levels of semantic distance. Thus, although we used neutral stimuli in the experiment, in order to ensure similarity to the design of Experiment 1, we excluded those stimuli from the statistical analyses.

Subjects. Ten students served as subjects, in partial fulfillment of course requirements. None of them had participated in Experiment 1.

\section{Results}

The error rates were very low. Subjects making numerical comparisons in their first session made $6 \%$ errors in incongruent trials and none in the congruent trials in the numerical comparison condition. These subjects showed the same error percentage (2\%) in congruent and incongruent physical comparison trials. When physical comparisons were made in the first session, subjects made 5\% errors in incongruent trials and none in congruent trials in the numerical comparison trials. These same subjects made $.5 \%$ errors in the incongruent trials and none in the congruent trials in the physical comparison condition. Although the percentages of errors were low, their trend fit in with the RT data. Mean RTs for the various experimental treatments are presented in Table 2.

The data were submitted to a four-way analysis of variance, with congruence, relevant dimension, and semantic distance as within-subjects factors and order of sessions as a between-subjects factor. This analysis was performed on the means of correct trials for each subject in each experimental condition. Congruent trials were $103 \mathrm{msec}$ faster than incongruent trials $[F(1,8)=75.62$, $\mathrm{p}<.001]$, physical comparisons were made $127 \mathrm{msec}$ faster than semantic comparisons $[F(1,8)=43.18$, $\mathrm{p}<.001$ ], and numerically distant stimuli (4-unit distance) were compared $21 \mathrm{msec}$ faster than numerically closer (2-unit distance) stimuli $[\mathrm{F}(1,8)=17.44$, $\mathrm{p}<.01]$. As in the previous experiment, the effect of congruence was a function of the type of comparison (relevant dimension) and order of sessions. This is evidenced in the second-order interaction $[F(1,8)=6.03$, $\mathrm{p}<.05]$ and in the interaction between relevant dimension and congruence $[F(1,8)=1,038.41, p<.001]$. Moreover, the effect of semantic distance depends both on relevant dimension and congruence. Both the secondorder interaction among semantic distance, relevant dimension, and congruence and the first-order interaction between semantic distance and relevant dimension were significant $[F(1,8)=8.39, p<.025, \quad F(1,8)=$ $33.01, \mathrm{p}<.001$, respectively]

Simple interaction effects between relevant dimensions and congruence were tested for each of the two orders of sessions. A significant interaction was found for those subjects who performed physical comparisons in their first session $[F(1,8)=187.60, p<.001]$, but no significant interaction effect was found for those who

Table 2

Mean Reaction Time (in Milliseconds) for Different Semantic Distances and Congruence Conditions Within Different Types of Comparisons

\begin{tabular}{lcccc}
\hline & \multicolumn{4}{c}{ Type of Comparison } \\
\cline { 2 - 5 } & \multicolumn{2}{c}{$\begin{array}{c}\text { First Session } \\
\text { Semantic }\end{array}$} & \multicolumn{2}{c}{$\begin{array}{c}\text { First Session } \\
\text { Physical }\end{array}$} \\
\cline { 2 - 5 } Congruence & Semantic & Physical & Semantic & Physical \\
\hline \multicolumn{4}{c}{ Two-Unit } & Distance \\
Congruent & 633 & 513 & 674 & 527 \\
Incongruent & 763 & 587 & 801 & 565 \\
& \multicolumn{4}{c}{ Four-Unit Distance } \\
Congruent & 591 & 489 & 614 & 528 \\
Incongruent & 671 & 657 & 742 & 607 \\
\hline
\end{tabular}




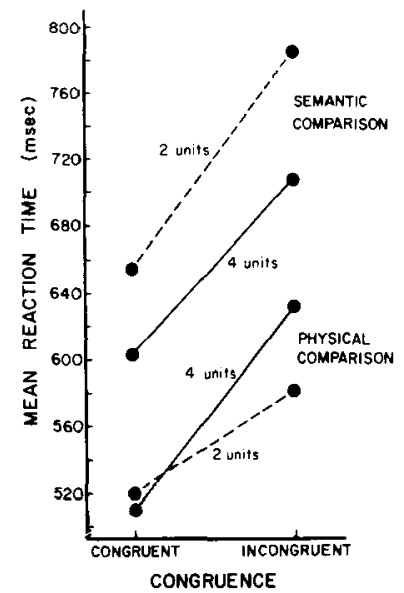

Figure 3. Mean reaction time in milliseconds as a function of congruence, relevant dimension, and semantic distance.

performed semantic comparisons in their first session. This pattern of results is similar to that found in the previous experiment.

A similar analysis was performed in the case of the interaction among congruence, semantic distance, and relevant dimension. When the comparisons were based on numerical differences, semantic distance had the same effect regardless of congruence $[F(1,8)=1.11]$. That is, the more distant (4-unit) pairs of digits were compared faster than the less distant (2-unit) pairs, for both congruent and incongruent trials. However, for physical size comparisons, the effect of semantic distance was dependent upon congruence level. The numerically distant pairs were compared $56 \mathrm{msec}$ slower than the numerically closer pairs when incongruent stimuli were compared $[F(1,8)=12.50, p<.01]$. In the congruent trials, the effect was not significant $(F<1)$ and was in the opposite direction. The relationship described by the second-order interaction, Congruence by Semantic Distance by Relevant Dimension, appears in Figure 3.

\section{Discussion}

The pattern of results regarding the congruence, relevant dimension, and order of sessions variables in the present experiment is very similar to that found in Experiment 1 . Namely, RTs for congruent trials were shorter than RTs for incongruent trials. Physical comparisons were made more rapidly than numerical comparisons. Physical size as an irrelevant dimension had more influence on performance than did semantic size as an irrelevant dimension. It was found that when physical size served as a relevant dimension in the first session, its influence on performance in the second session (as irrelevant dimension) was stronger (stronger congruence effect) than the parallel influence of the numerical size. The order of the effects here is the same as in the previous experiment.

The major contribution of the present experiment is the interaction among congruence, relevant dimension, and numerical distance. When comparisons are based upon numerical size, the effects of the numerical distance and congruence are additive (replicating Banks', 1977, results), whereas these two variables interact when physical size serves as relevant dimension. An additional analysis revealed that on incongruent trials, stimuli with smaller distance are compared faster than stimuli with larger (4-unit) distance. On congruent trials, the effect is nonsignificant and in the opposite direction. In terms of the additive factors logic, it seems that size congruity and numerical distance effects are not additive and therefore do not emerge from independent processing stages. The data suggest that physical and numerical differences are extracted in parallel by the system.

\section{GENERAL DISCUSSION}

In both experiments, we have found that RT is influenced by the degree of correspondence (i.e., congruence) between relevant and irrelevant dimensions. Moreover, this is true not only when the faster process (physical comparison) is irrelevant, but also when the slower process (numerical comparison) is irrelevant. Thus, it is reasonable to conclude that information about physical and numerical differences accrues in parallel.

When numerical distance is manipulated, it has an effect even when irrelevant to the task. Moreover, it interacts with congruence when comparisons of physical size are made. Hence, numerical distances are automatically computed by the system in this task, and they affect relevant judgment. Since numerical values affect physical judgments, it is necessary to suppose that physical information and numerical information are handled in parallel at some stage of the system. It is possible that the two processes are separate but that their outputs interfere or that the two are not kept separate by the system (i.e., there is some cross talk between them during processing).

An interesting result in both experiments is the effect of order of sessions on performance. We have already noted that the size congruity effect is stronger when physical size rather than semantic size serves as an irrelevant dimension. This disparity in the effects of the two dimensions is more salient for subjects who performed physical comparisons in their first session than for subjects who worked in the reverse order. We did not expect such a result. However, we would like to suggest an ad hoc explanation for it. It is possible that experience with comparisons based upon a certain dimension sensitizes use of these comparisons. This line of thinking suggests that short-term practice might vary the degree to which irrelevant information is processed and can be a source of interference.

As mentioned earlier, Banks (Banks, 1977; Banks \& Flora, 1977) suggests that the size congruity effect emerges at an early stage of processing. This notion is based upon two results reported by Paivio (1975). 
The size congruity effect is evidenced for pictures but not for words, and pictures are compared faster than words. We have found an effect of a slower process (numerical) on a faster one (physical) and have suggested parallel rather than sequential processing. If the rate differences between two processes do not limit interference effects, one might wonder why the effect is limited to one set of stimuli in Paivio's experiment. Besner and Coltheart (1979) suggest an answer. They report results similar to those of Paivio with numerals instead of animals and objects, that is, a size congruity effect for Arabic numerals but not for alphabetically written digits (e.g., "one"). Besner and Coltheart suggest that the difference between the two sets of stimuli lies in the different mechanisms employed in the two tasks. Pictures and Arabic numerals are processed ideographically, whereas numbers represented in script are processed alphabetically. This notion gains support from neuropsychological studies of alexic patients. Hécaen (1979) describes a version of alexia without agraphia (the patient manifests a reading disorder without a deficit in writing), in which patients are unable to read letters or words despite the relatively normal ability to read single- and multiple-digit numbers. Hecaen and Kremin (1976) report four cases of such patients who were better at reading aloud Arabic numerals than at reading words.

\section{REFERENCE NOTES}

1. Hinrichs, J. V. Physical and numerical size in number comparisons. Paper presented at the annual meeting of the Psychonomic Society, November 1976.

2. Yurko, D. S., \& Hinrichs, J. V. Judgment of numerical inequality: Size-value congruity. Paper presented at the meeting of the Midwestern Psychological Association, Chicago, May 1978.

\section{REFERENCES}

Banks, W. P. Encoding and processing of symbolic information in comparative judgments. In G. H. Bower (Ed.), The psychol- ogy of learning and motivation (Vol. 11). New York: Academic Press, 1977.

Banks, W. P., \& FlorA, J. Semantic and perceptual processes in symbolic comparisons. Journal of Experimental Psychology: Human Perception and Performance, 1977, 3, 278-290.

Besner, D., \& Coltheart, M. Ideographic and alphabetic processing in skilled reading of English. Neuropsychologia, $1979,17,467-472$.

Dixon, P., \& Just, M. A. Normalization of irrelevant dimensions in stimulus comparisons. Journal of Experimental Psychology: Human Perception and Performance, 1978, 4, 36-46.

HÉCAEn, H. Aphasias. In M. S. Gazzaniga (Ed.), Handbook of behavioral neurobiology (Vol. 2). New York: Plenum, 1979.

HÉCaen, H., \& Kremin, H. Neurolinguistic research on reading disorders resulting from left hemisphere lesions. In $\mathrm{H}$. Whitaker \& H. A. Whitaker (Eds.), Studies in neurolinguistics (Vol. 2). New York: Academic Press, 1976.

HoLYOAK, K. J. The form of analog size information in memory. Cognitive Psychology, 1977, 9, 31-51.

MOYER, R. S. Comparing objects in memory: Evidence suggesting an internal psychophysics. Perception \& Psychophysics, 1973, 13, 180-184.

Moyer, R. S., Bradley, D. R., Sorensen, M. H., Whiting, J. C., \& Mansfield, D. P. Psychophysical functions for perceived and remembered size. Science, 1978, 200, 330-332.

Paivio, A. Perceptual comparisons through the mind's eye. Memory \& Cognition, 1975, 3, 635-647.

STERnBe RG, $\mathbf{S}$. The discovery of processing stages: Extension of Donder's method. Acta Psychologia, 1969, 30, 276-315.

\section{NOTES}

1. These references were brought to our attention after the first version of the manuscript was already written.

2. It is possible that the small difference between neutral and congruent trials in the physical comparisons is due to the nature of the stimuli. Neutral stimuli for physical comparisons are identical digits (e.g., 3 3). This could create a special advantage for these trials and accelerate their processing.

3. These combinations of physical sizes were used in order that all the physical heights $(4,5,6 \mathrm{~mm})$ would be equally likely and in order to include two physical differences in the present experiment (instead of one in Experiment 1).

(Received for publication May 1, 1981; revision accepted March 19, 1982.) 